\title{
Síndrome Cardiorrenal tipo II: bases fisiopatológicas e terapêuticas em cães e gatos
}

\author{
[Cardiorenal Syndrome type II: physiopathological and therapeutic bases in dogs and cats]
}

\section{"Revisão/Review"}

\author{
Aline Nochi Berto*, Beatriz Teixeira Martuchi, Luiz Otávio Rodrigues Ribeiro, Rodrigo \\ Prevedello Franco
}

Universidade de Marília (UNIMAR), Marília-SP, Brasil.

*Autor para correspondência/Corresponding author: E-mail: aline_nochi@hotmail.com

\begin{abstract}
Resumo
A Síndrome Cardiorrenal (SCR) do tipo II é reconhecida por anormalidades crônicas da função cardíaca que promove lesão e disfunção renal. Nessa revisão de literatura será discutida a compreensão atual, mecanismos fisiopatológicos, aspectos etiológicos e clínicos, bem como a adequação terapêutica de cães e gatos acometidos pela síndrome cardiorrenal do tipo II. Devido à complexidade da síndrome, refletir sobre os diversos mecanismos fisiopatológicos torna-se essencial na continuidade de pesquisas em cães e gatos relacionadas ao diagnóstico precoce e, principalmente, pontos importantes a serem enfatizados no tratamento, com o intuito de obter melhores resultados na terapia de pacientes cardiorrenais.
\end{abstract}

Palavras-chave: insuficiência cardíaca; insuficiência renal; doença valvar crônica.

\begin{abstract}
The Cardiorenal Syndrome (CRS) Type II is recognized by chronic abnormalities of the cardiac function which promotes lesion and renal dysfunction. In this literature review we are going to discuss the actual comprehension, pathophysiological mechanisms, etiological aspects and clinical, such as therapeutic adequacy of dogs and cats affected by the cardiorenal syndrome type II. Due to the complexity of the syndrome, reflecting over several pathophysiological mechanisms has become essential in continuing researches in dogs and cats, related to the early diagnosis and especially emphasizing important points in treatment, with the intention of obtaining the best results in therapy of cardiorenal patients.
\end{abstract}

Keywords: cardiac insufficiency; renal insufficiency; chronic valvular disease.

\section{Introdução}

O sistema cardiovascular possui a importante função de manutenção da pressão arterial e débito cardíaco, visando a manutenção da perfusão e oxigenação tecidual orgânica. A função renal, dentre suas várias funções, desempenha um papel essencial na manutenção da homeostase através da reabsorção ou excreção de substâncias filtradas nos glomérulos, da regulação de íons e eletrólitos, bem como da pressão arterial. Assim, existe uma estreita relação entre os sistemas e quando há uma disfunção em ambos, esta é denominada de Síndrome Cardiorrenal (SCR) (Jois e Mebazaa, 2012).
Em 2010, em uma conferência médica no âmbito do Acute Dialysis Quality Initiative (ADQI), foi adotado o termo SCR como "Distúrbios do coração e dos rins em que a disfunção aguda ou crônica em um órgão é capaz de induzir a disfunção aguda ou crônica no outro"; e a subdividiram em cinco subtipos conforme a etiologia primária (Tabela 1). A SCR do tipo II é reconhecida por anormalidades crônicas na função cardíaca que acarreta em lesão e disfunção renal, com Degeneração Mixomatosa Valvar Mitral e/ou Tricúspide Crônica (DVMT) nos cães e a Cardiomiopatia Hipertrófica (CMHF) nos felinos, responsáveis pelo agravamento da Insuficiência 
Cardíaca Congestiva (ICC) e principal indutora da SCR (Ronco et al., 2010; Freitas et al., 2014).

As terapêuticas implementadas no controle da ICC visam ao aumento da sobrevida dos pacientes, no entanto, podem potencializar o aparecimento da SCR. Diante do apresentado, a revisão de literatura tem como objetivo discutir os mecanismos fisiopatológicos, aspectos clínicos e terapêuticos da síndrome cardiorrenal do tipo II em cães e gatos (Mora et al., 2016).

Tabela 1. Subdivisão dos Tipos da Síndrome Cardiorrenal proposta por Ronco et al. (2010).

\begin{tabular}{cl}
\hline Tipo da SCR & Sinais \\
\hline SCR Tipo I (Síndrome Cardiorrenal Aguda) & $\begin{array}{l}\text { Piora abrupta da função cardíaca (por exemplo, insuficiência } \\
\text { cardíaca congestiva agudamente decompensada) levando à lesão } \\
\text { renal aguda } \\
\text { Anormalidades crônicas na função cardíaca (por exemplo, } \\
\text { SCR Tipo II (Síndrome Cardiorrenal crônica) } \\
\text { insuficiência cardíaca congestiva crônica) causando doença } \\
\text { renal crônica progressiva e permanente }\end{array}$ \\
PCR Tipo III (Síndrome Renocardíaca Aguda) & $\begin{array}{l}\text { Piorupta da função renal (por exemplo, lesão renal aguda) } \\
\text { causando distúrbio cardíaco agudo (insuficiência cardíaca }\end{array}$ \\
aguda) & $\begin{array}{l}\text { Doença renal crônica (por exemplo, doença glomerular crônica) } \\
\text { contribuindo para a diminuição da função cardíaca e hipertrofia } \\
\text { ventricular }\end{array}$ \\
& $\begin{array}{l}\text { Existência de doença sistêmica (por exemplo, sepse) causando } \\
\text { lesão renal e cardíaca aguda simultaneamente }\end{array}$ \\
\hline
\end{tabular}

(Ronco et al., 2010).

\section{Definição e Etiologia}

A insuficiência cardíaca é reconhecida como uma síndrome clínica, advinda da disfunção cardíaca estrutural ou funcional, que resulta na redução do enchimento ventricular ou falha da ejeção sanguínea, resultando na ativação de mecanismos compensatórios (Chawla et al., 2014).

A DVMT, cardiopatia com maior incidência na rotina da clínica médica $(75 \%$ dos casos), é um processo degenerativo crônico e progressivo das valvas atrioventriculares com importante disfunção ventricular e quadros de ICC em cães, acometendo principalmente as raças de pequeno a médio porte (Chamas et al., 2011).

A segunda afecção cardíaca mais frequente é a cardiomiopatia dilatada canina, caracterizada por importante disfunção sistólica ventricular progressiva em cães de grande porte (Dutton e López-Alvarez, 2018).

Nos gatos, a CMHF é caracterizada inicialmente pela disfunção diastólica e posteriormente pela disfunção sistólica ventricular esquerda, com a ocorrência de 57,5\% ( $\mathrm{n}=106)$, seguida pela cardiomiopatia restritiva $(20,7 \%)$, cardiomiopatia dilatada felina $(10,4 \%)$ e a cardiomiopatia não classificada $(10,4 \%)$ (Ferasin et al., 2003).

As cardiopatias congênitas também levam à ICC em cães e gatos, porém apresentam uma menor incidência quando comparadas às cardiopatias adquiridas (Oliveira et al., 2011; Tidholm et al., 2015). Entretanto, todas as cardiopatias podem evoluir com a redução do débito cardíaco e ativação dos mecanismos compensatórios, fatos que resultam na redução do fluxo sanguíneo renal, de forma aguda ou crônica, induzindo a injúrias agudas, como Insuficiência Renal Aguda (IRA) e Doenças Renais Crônicas (DRC) (Shah e Greaves, 2011).

\section{Mecanismos Fisiopatológicos}

A base fisiopatológica da SCR é explicada pela redução do débito cardíaco causado pela ICC crônica (Shrestha et al., 2010). A ativação dos sistemas neuro-humorais como o Sistema Nervoso Simpático (SNS), Sistema Renina Angiotensina Aldosterona (SRAA), o aumento da pressão arterial, a inflamação celular, a geração de Espécies Reativas de Oxigênio (EROs) e a diminuição de óxido nítrico (NO) são determinantes na interação e disfunção cardiorrenal (Martins et al., 2011).

O SNS apresenta um importante papel na progressão da SCR devido à ativação dos barorreceptores arteriais ao reconhecer a diminuição da pressão arterial e débito cardíaco, promovendo o aumento da frequência e da contratilidade miocárdica, bem como a vasoconstrição arterial e venosa (Medeiros et al., 
2009). A finalidade é melhorar o aporte sanguíneo de órgãos vitais em função da vasoconstrição periférica de órgãos não vitais, como os rins. Fato que reduz a taxa de filtração glomerular e a ativação do sistema SRAA, que agrava o quadro congestivo e contribui para a progressão da SCR (Palazzuoli e Ronco, 2011).

Nos rins, a hipertensão arterial agrava a função renal, como evidenciado pelo estudo de Grauer et al. (2009) em cães e gatos portadores de DRC, que observaram que a elevação da pressão arterial é diretamente proporcional ao aumento da proteinúria, e resulta em diminuição da taxa de filtração glomerular.

A congestão venosa promovida pelo SRAA também foi considerada fator determinante na progressão da ICC e da SCR devido ao aumento na Pressão Venosa Central (PVC), resultando na hipertensão dos túbulos distais e veias renais, disfunção tubular, proteinúria e hipóxia do parênquima renal (Jois e Mebazaa, 2012).

Um dos pontos importantes da SCR em relação ao SRAA é o estresse oxidativo e seus mecanismos inflamatórios, com a redução do óxido nítrico na corrente sanguínea. As EROs são capazes de lesionar células da musculatura lisa, células tubulares renais, células do endotélio vascular e cardiomiócitos, provocando inflamação e disfunção progressiva dos órgãos (Mora et al., 2016). Além disso, reduz o óxido nítrico, um potente vasodilatador e natriurético que interfere na frequência cardíaca e na respiração mitocondrial (metabolismo energético), induzindo a hipertrofia e apoptose dos miócitos (Sarraf et al., 2009).

Outra condição importante a ser considerada é o desenvolvimento da anemia em pacientes com SCR. Foi demonstrado que a associação entre insuficiência cardíaca, insuficiência renal e anemia pode contribuir para o desenvolvimento e piora de cada uma dessas condições (Fiocchi et al., 2017). Yu e Huang (2016), quando estudaram a prevalência da anemia em 114 cães com DVMT, descobriram que a concentração da hemoglobina e do hematócrito diminuíram com o avanço da classe funcional da ICC nos cães acometidos. Em pacientes com DRC, há evidências de que a anemia está associada à progressão da doença devido à diminuição do fluxo sanguíneo e do fornecimento de oxigênio aos rins, além da indução do estresse oxidativo (Bartges, 2012).
A etiologia da anemia na SCR é multifatorial, incluindo a redução da síntese de eritropoietina, o aumento das citocinas próinflamatórias que produzem depressão da medula óssea e a redução da absorção intestinal de ferro causada por hiporexia ou edema nas alças intestinais (Souza et al., 2010).

\section{O Diagnóstico SCR Tipo II}

As estreitas interrelações entre os sistemas cardiorrenais devem ser levadas em consideração no momento do diagnóstico e manejo de pacientes com a SCR, devido às comorbidades de eventos da ICC e DRC. O hemograma, o perfil bioquímico sérico, a urinálise, a mensuração da pressão arterial, radiografia torácica e ultrassonografia abdominal, além de eletrocardiografia e a ecocardiografia, são ferramentas diagnósticas necessárias (Pouchelon et al., 2015).

O estadiamento da ICC e da DRC é essencial para a elaboração de planos terapêuticos subsequentes (Orvalho e Cowgill, 2017). Para o estadiamento da ICC, são bem aceitas as classificações baseadas no American College of Veterinary Internal Medicine (ACVIM) (Tabela 2) (Atkins et al., 2009), para cães com DVMT, e a International Small Animal Cardiac Health Council (ISACHC) apresentada na Tabela 3, indicada para cães e gatos com as demais cardiopatias.

No entanto, no contexto da síndrome cardiorrenal, o ISACHC ou sistemas similares são considerados, isoladamente, meios incompletos para categorizar o envolvimento do sistema cardiovascular, pois falham em explicar importantes anormalidades cardiovasculares que podem se desenvolver na DRC, incluindo hipertensão arterial sistêmica, disfunção diastólica e sistólica, arritmias e alterações em biomarcadores (Pouchelon et al., 2015). Desta forma, é necessária a utilização de outras potenciais ferramentas para diagnóstico e estadiamento precisos, que auxiliem no planejamento de planos terapêuticos subsequentes.

No diagnóstico das cardiopatias, os exames de imagem, como a radiografia torácica em duas projeções ortogonais, visam avaliar o remodelamento cardíaco, o padrão parenquimatoso pulmonar e estruturas vasculares, considerados indicadores de cardiopatias e evolução para ICC (Pouchelon et al., 2015). O eletrocardiograma (ECG) é uma ferramenta 
importante no diagnóstico de arritmias cardíacas e distúrbios de condução. Estudos eletrofisiológicos experimentais em ratos portadores de DRC evidenciaram aspectos cardíacos arritmogênicos, como prolongamento do potencial de ação, despolarizações ventriculares precoces e frequentes, instabilidade elétrica e maior sensibilidade à fibrilação ventricular (Hsueh et al., 2014).

Tabela 2. Classificação da American College of Veterinary Internal Medicine (ACVIM).

\begin{tabular}{cll}
\hline & Classe & Características do doente \\
\hline A & B1 & $\begin{array}{l}\text { Cães em risco de desenvolver doença cardíaca. } \\
\text { Cães assintomáticos: sem remodelação cardíaca } \\
\text { Cães assintomáticos: com remodelação } \\
\text { cardíaca } \\
\text { B2 }\end{array}$ \\
C & C Agudo & $\begin{array}{l}\text { Insuficiência cardíaca congestiva } \\
\text { descompensada - requer hospitalização }\end{array}$ \\
& C Crônico & $\begin{array}{l}\text { Insuficiência cardíaca congestiva compensada - } \\
\text { ambulatório } \\
\text { Insuficiência cardíaca congestiva refratária ao } \\
\text { tratamento padrão - requer hospitalização }\end{array}$ \\
& D Agudo & $\begin{array}{l}\text { Insuficiência cardíaca congestiva refratária ao } \\
\text { tratamento padrão - ambulatório }\end{array}$ \\
\hline
\end{tabular}

American College of Veterinary Internal Medicine (ACVIM) (Atkins et al., 2009).

Tabela 3. Classificação da International Small Animal Cardiac Health Council (ISACHC).

\begin{tabular}{ll}
\hline Classe & Descrição da IC \\
\hline Classe I & $\begin{array}{l}\text { Assintomática: doença cardíaca detectável, } \\
\text { mas sem a presença de sinais clínicos de } \\
\text { insuficiência cardíaca. }\end{array}$ \\
Classe II & $\begin{array}{l}\text { Leve a moderada: Sinais clínicos de } \\
\text { insuficiência cardíaca presentes em repouso } \\
\text { ou com exercícios mínimos. Os sinais } \\
\text { clínicos incluem dispneia leve, intolerância } \\
\text { ao exercício, taquipneia e ascite. }\end{array}$
\end{tabular}

Classe III Avançada: Sinais clínicos de doença cardíaca grave mesmo em repouso, com dispneia acentuada, intolerância ao exercício e hipoperfusão. Os casos mais graves Subclassificacão

IA: sem evidências de compensação
(sobrecarga de pressão ou volume)

IB: com sinais de compensação detectados por radiografia ou ecocardiografia apresentam choque cardiogênico.

International Small Animal Cardiac Health Council (ISACHC) (Bonagura et al., 1995).

A ecocardiografia é um exame fundamental para a avaliação da anatomia e função miocárdica, velocidade e direção de fluxos sanguíneos. Suas variáveis determinam a cronicidade e a extensão do remodelamento cardíaco, progressão da disfunção miocárdica, redução do débito cardíaco, e predizem a mortalidade e longevidade do paciente, bem como auxiliam na caracterização da ICC e no manejo terapêutico (Oliveira et al., 2017).

Algumas variáveis ecocardiográficas, como o Diâmetro Diastólico Final do Ventrículo Esquerdo (DIVEd), a relação Átrio Esquerdo e Raiz Aórtica (AE/Ao), os valores de fração de ejeção e de encurtamento, a distância do ponto $E$ da Valva Mitral ao Septo (SSPE) e o movimento anular de mitral, o Tempo de Relaxamento Volumétrico (TRIV/ms), a velocidade da onda $\mathrm{E}$ $(\mathrm{m} / \mathrm{s})$ e onda A (m/s) e sua relação (E/A) do fluxo transmitral, a relação E:TRIV e o Doppler Tecidual Miocárdico (TDI) são preditoras do agravamento da ICC ou da mortalidade em cães e gatos cardiopatas (Oliveira et al., 2017; Strunz et al., 2017).

Em relação aos biomarcadores nas cardiopatias, os peptídeos natriuréticos do tipo NTerminal do pro-B (NT-proBNP), o Peptídeo Natriurético do tipo B (BNP), o Peptídeo Natriurético Pro-atrial N-terminal (NT-proANP) e a Troponina Cardíaca I (cTnI) são utilizados 
visando identificar e avaliar a progressão da disfunção ventricular e atrial na ICC em cães e gatos (Pouchelon et al., 2015).

Em relação ao estadiamento da DRC, recomenda-se a classificação da IRIS encontra-se na Tabela 5, e por fim, na hipertensão arterial sistêmica representada na Tabela 6 (Bonagura et al., 1995; IRIS, 2013). Ao contrário dos sistemas de estadiamento cardiovascular existentes, as diretrizes IRIS para doenças renais foram consideradas
(International Renal Interest Society) (Orvalho e Cowgill, 2017) baseado nos valores séricos de creatinina, que estão apresentos na Tabela 4, na relação Proteína/Creatinina Urinária (UPC) que

suficientemente completas para a descrição de lesões renais na síndrome cardiorrenal (Pouchelon et al., 2015). Os sistemas IRIS abrangem uma avaliação de danos leves a graves, incluindo vários índices importantes da função renal.

Tabela 4. Estadiamento IRIS em relação à creatinina sérica.

\begin{tabular}{ccl}
\hline Estágio & $\begin{array}{c}\text { Concentração de creatinina } \\
\text { sérica }(\mathbf{m g} / \mathbf{d L})\end{array}$ & \multicolumn{1}{c}{ Comentários } \\
\hline 1 & $<1,5$ cães & $\begin{array}{l}\text { Sem azotemia. Inabilidade na concentração } \\
\text { urinária. Hipertensão arterial. Proteinúria. }\end{array}$ \\
2 & 1,6 gatos & Azotemia leve. Sinais brandos ou ausentes. \\
1,6 a 2,8 gatos & 2,1 a 5 cães & Azotemia moderada. Sinais sistêmicos \\
& 2,9 a 5 gatos & persistentes. \\
& $>5$ cães e gatos & $\begin{array}{l}\text { Azotemia severa. Risco aumentado de sinais } \\
\text { sistêmicos e crise urêmica. }\end{array}$ \\
\hline
\end{tabular}

International Renal Interest Society, 2013.

A creatinina sérica é o principal marcador de filtração glomerular, com ressalva nos casos de lesão tubular e ausência de redução significativa da TFG (Palazzuoli et al., 2015). Fato que justifica valores normais de creatinina até que $66 \%$ a $75 \%$ dos néfrons fiquem afuncionais (Freitas et al., 2014). Assim, a busca por novos biomarcadores precoces é necessária e pode influenciar nas intervenções terapêuticas cardiovasculares (Orvalho e Cowgill, 2017).

Tabela 5. Substadiamento IRIS em relação à proteinúria.

\begin{tabular}{ccc}
\hline \multicolumn{2}{c}{$\begin{array}{c}\text { Relação } \\
\text { proteína/creatinina } \\
\text { urinária }\end{array}$} & Classificação \\
\hline \multicolumn{2}{c}{ Cães } & Gatos \\
$<0,2$ & $<0,2$ & Não proteinúrico \\
$0,2-0,5$ & $0,2-0,4$ & Limítrofe proteinúrico \\
$>0,5$ & $>0,4$ & Proteinúrico \\
\hline International Renal Interest & Society, 2013.
\end{tabular}

Recentemente, a cistatina $\mathrm{C}$, a lipocalina associada à Gelatinase Neutrofílica (NGAL), a Nacetil- $\beta$-D-glucosaminidase (NAG) e a enzima Gama-Glutamiltransferase (GGT) urinária foram avaliadas em pacientes com ICC e seus níveis plasmáticos apresentaram propriedades prognósticas na SCR (Freitas et al., 2014; Ronco e
Di Lullo, 2017). Em um estudo clínico com cães, a NGAL urinária foi identificada em pacientes com desenvolvimento de IRA, doze horas antes do aumento da creatinina ou NGAL sérico (Lee et al., 2012).

Segundo Hall et al. (2016), a Dimetilarginina Dimétrica (SDMA), um biomarcador renal recém descoberto, foi considerado um marcador endógeno da TFG adequado para identificar IRA e DRC em cães e gatos, não sendo influenciado pela massa muscular como a creatinina sérica. No mesmo estudo, os autores demonstraram que o SDMA sérico eleva-se aproximadamente 10 meses antes da creatinina em cães com DRC. No contexto de SCR, novos estudos com o SDMA em pacientes cardiopatas podem auxiliar no diagnóstico precoce da SCR tipo II e no ajuste terapêutico de pacientes portadores de ICC avançada.

Como o rim e o coração são dois órgãos em risco de danos devido à hipertensão arterial sistêmica, e como a doença renal é freqüentemente associada à hipertensão arterial sistêmica, a pressão arterial deve ser monitorada sistematicamente nas doenças renais e cardiovasculares (Pouchelon et al., 2015).

No que diz respeito ao sistema renal, a hipertensão arterial sistêmica pode resultar em 
aumento da taxa de lesão renal e proteinúria (Jacob et al., 2003). A elevação da relação UPC, um marcador precoce e de prognóstico na DRC de cães e gatos, correlaciona-se com a progressão da doença renal e está associada aos índices de mortalidade (Rossi et al., 2016). Em relação ao sistema cardiovascular, a alteração mais comum associada à hipertensão em cães e gatos é a hipertrofia concêntrica do ventrículo esquerdo (HVE) (Acierno et al., 2018).

Tabela 6. Substadiamento IRIS em relação à pressão arterial.

\begin{tabular}{cc}
\hline $\begin{array}{c}\text { Pressão arterial } \\
\text { sistêmica }\end{array}$ & Classificação \\
\hline$<150 \mathrm{mmHg}$ & Risco mínimo \\
150 a $159 \mathrm{mmHg}$ & Risco baixo \\
160 a $179 \mathrm{mmHg}$ & Risco moderado \\
$>180 \mathrm{mmHg}$ & Risco severo \\
\hline
\end{tabular}

International Renal Interest Society, 2013.

A urinálise é um teste laboratorial simples e não invasivo que pode conceder informações relevantes mesmo no paciente assintomático. A urinálise pode demonstrar proteinúria, baixa densidade urinária, cilindrúria, glicosúria, hematúria e alteração no $\mathrm{pH}$ urinário. O sedimento urinário pode evidenciar a elevação do número de leucócitos, hemácias, células epiteliais renais e cilindros granulosos ou céreos (Freitas et al., 2014).

Os rins são responsáveis por manter a homeostase no corpo; logo, a insuficiência renal normalmente leva a alterações de eletrólitos e equilíbrio ácido-base (Langston, 2017). A hipocalemia sérica decorre da hiporexia e a excessiva perda gastrintestinal ou urinária, a hiponatremia ocorre pela redução na absorção dos túbulos renais e a hiperfosfatemia resulta da redução da Taxa de Filtração Glomerular (TFG), estando associada à hipocalcemia e as baixas concentrações de calcitriol sérico. Por fim, a hipocloremia está associada aos distúrbios ácidobásicos e correlacionada com a acidose metabólica, com pH sérico menor que 7,1 e concentrações de $\mathrm{HCO}_{3}$ - inferiores a $8 \mathrm{mEq} / \mathrm{L}$. Estas complicações agravam a DRC e compremetem também a função sistólica ventricular, devendo desta forma, ser monitoradas e corrigidas (Polzin et al., 2013).

As anormalidades morfológicas renais são avaliadas pela ultrassonografia abdominal (Pouchelon et al., 2015), onde pacientes com DRC apresentam redução da espessura da cortical, perda da relação corticomedular, aumento da ecogenicidade parenquimatosa (Ronco e Di Lullo, 2017), e redução do fluxo arterial intra-renal calculado pelo Índice de Resistividade (IR) e o Índice de Pulsatilidade (PI). Esses valores quando aumentados são úteis na identificação da nefropatia quando há ausência de demais alterações ultrassonográficas no modo bidimensional ou quando apenas o aumento na ecogenicidade cortical é observado (Bragato et al., 2017).

\section{Tratamento da SCR Tipo II}

As terapias atuais recomendadas para ICC demonstraram melhorar a qualidade de vida e a sobrevivência dos pacientes, entretanto podem comprometer a função renal (Grande et al., 2017).

Com o estadiamento da ICC, pacientes da classe Ib (ISACHC) e B2 avançado (ACVIM) já necessitam de terapia farmacológica, e em classes mais avançadas (classes II e III / ISACHC ou C e D / ACVIM), onde os pacientes são portadores de sinais clínicos ou edema pulmonar cardiogênico. Fármacos como furosemida, torasemida, espironolactona e hidroclorotiazida, inibidores da ECA, amlodipina, hidralazina, digoxina e pimobendan, além de antiarrítmicos, venodilatadores, antitrombóticos e antioxidantes são utilizados em associação, visando ao controle do quadro da ICC (Atkins et al., 2002; Pouchelon, 2015).

Nos estágios mais avançados da doença cardíaca, o débito cardíaco, a fração de ejeção e a fração de encurtamento ventricular esquerda reduzem de forma considerável, diminuindo a perfusão renal e induzindo a DRC, levando à perda da capacidade renal em manter a taxa de filtração glomerular, o controle hidroeletrolítico e ácido-básico, além do acúmulo de substâncias tóxicas do organismo (Freitas et al., 2016).

Nos pacientes com SCR a fluidoterapia deve ser monitorada, objetivando a manutenção do volume circulatório, do débito cardíaco e da perfusão sistêmica, atentando-se para o aparecimento de sinais clínicos relacionados à sobrecarga de volume, como taquipneia, dispneia, edema periférico e estertores pulmonares (Davis et al., 2013; Pouchelon, 2015).

Os fluidos são administrados conforme a necessidade do paciente, controlando volume e taxa de reposição necessários. De acordo com a American Animal Hospital Association (AAHA), a solução de Cloreto de Sódio a 0,45\% com 
dextrose a $2,5 \%$ é o fluido de escolha devido a menor concentração de sódio (Davis et al., 2013). A taxa de infusão depende da condição clínica do paciente, no entanto, a reposição rápida de volume (0 a 6 horas) pode agravar a ICC, sendo recomendado prolongar o tempo de infusão para seis a doze horas. Após reposição inicial, o volume de manutenção administrado é calculado por $66 \mathrm{~mL} / \mathrm{kg} / \mathrm{dia}$, contando a perda de volume contínua por vômito e diarreia urêmica e perdas insensíveis, como os fluidos perdidos através da respiração e fezes normais (Langston, 2017).

A prática de induzir a diurese administrando mais fluido do que o necessário tem sido defendida há muito tempo para "eliminar toxinas urêmicas", contudo é improvável que a abordagem melhore a função renal na maioria dos casos, pois a promoção da sobrecarga de fluidos piora a oferta de oxigênio nos rins (Prowle e Bellomo, 2013).

Os diuréticos de alça são amplamente utilizados para o controle da sobrecarga de volume na ICC, com a dose diária da furosemida para cães variando de $1-2 \mathrm{mg} / \mathrm{kg}$ a cada 12 horas a 4-6 mg/kg a cada 8 horas, por via oral (Atkins et al., 2009). Contudo, o seu uso prolongado pode exercer efeitos negativos sobre a função renal, como a hipovolemia e a consequente estimulação do SRAA e SNS. As altas dosagens que controlam os sinais congestivos devem ser fortemente desencorajadas em função de seus efeitos colaterais, como hipocalemia, hipotensão, ativação neuro-hormonal acentuada e possível comprometimento renal (Ronco e Di Lullo, 2017).

A resistência aos diuréticos de alça deve ser considerada no tratamento da SCR, necessitando a associação de outros grupos diuréticos como a hidroclorotiazida (2-4 mg/kg, a cada 12 ou 24 horas, por via oral) (Feijó et al., 2016). Contudo, essa associação causa sinergismo e pode levar a intensa depleção de volume e até mesmo desequilíbrios eletrolíticos, como hipocalemia e hipomagnesemia, elevando-se o risco de arritmias (Atkins et al., 2009).

A torasemida (recomendada na dose diária de $0,1 \mathrm{mg} / \mathrm{kg}$ ), um diurético de alça, possui 12 horas de ação e meia-vida de 120 minutos após a administração Intravenosa (IV). Além de produzir uma ação diurética duradoura e potente, apresenta também ações benéficas de propriedades vasodilatadoras que auxiliam na redução do remodelamento cardíaco. Seus efeitos antifibróticos no miocárdico são similares aos mediados pelo antagonismo da aldosterona (Peddle et al., 2012).

\section{Distúrbios Eletrolíticos e Ácido-base}

Além dos distúrbios eletrolíticos causados por efeitos colaterais de fármacos no tratamento da ICC, há também a incapacidade dos rins na manutenção do equilíbrio eletrolítico devido à DRC. Dentre as principais alterações eletrolíticas estão a hipocalemia, que gera sinais clínicos de fraqueza muscular e letargia; e a hiperfosfatemia e a hipocalcemia, ambas capazes de promover o hiperparatireoidismo secundário renal (Polzin, 2013).

Para a reposição de potássio por via oral indica-se o gluconato de potássio $(2 \mathrm{mEq} / \mathrm{kg}$, a cada 12 horas, por via oral) ou citrato de potássio (75 mg/kg, a cada 12 horas, por via oral) (Bartges, 2012). Para minimizar a concentração sérica de fósforo, indica-se a utilização dos quelantes de fósforo (hidróxido de alumínio, carbonato de alumínio e óxido de alumínio) na dose inicial de 30-100 mg/kg/dia para cães e gatos. Já para correção da hipocalcemia, recomenda-se a administração de calcitriol na dose inicial de 2,0$2,5 \mathrm{ng} / \mathrm{kg}$, a cada 24 horas, por via oral (Polzin, 2013).

A acidose metabólica é um quadro comum na DRC, responsável pela ocorrência de vômito, anorexia, letargia, perda muscular (devido ao catabolismo muscular) e perda de peso. Agentes alcalinizantes orais podem ser usados na correção da acidose metabólica, como o bicarbonato de sódio na dosagem de $8-12 \mathrm{mg} / \mathrm{kg}$, administrado por via oral, a cada 8 ou 12 horas, e o citrato de potássio na dosagem de $40-75 \mathrm{mg} / \mathrm{kg}$, a cada 12 horas, por via oral (Langston, 2017).

\section{Controle da Hipertensão Arterial}

Em cães e gatos com SCR recomenda-se a manutenção da pressão arterial sistólica em valores menores que $160 \mathrm{mmHg}$, com o intuito de prevenir ou minimizar os danos causados a órgãos-alvo, como o coração e os rins (Pouchelon et al., 2015). Em cães, a terapêutica de primeira escolha é o grupo dos iECAs, como o Maleato de Enalapril $(0,5 \mathrm{mg} / \mathrm{kg}$, a cada 12 horas $)$ ou o Benazepril $(0,25-0,5 \mathrm{mg} / \mathrm{kg}$, a cada 24 horas). Em felinos, recomenda-se iniciar a terapia com amlodipina $(0,125-0,25 \mathrm{mg} / \mathrm{kg}$, a cada 24 horas), um fármaco da classe dos Bloqueadores de Canais de Cálcio (Feijó et al., 2016). 
Deve haver cautela na instituição das doses de iECAs em cães e gatos desidratados, uma vez que a diminuição da TFG é vigorosa nestes casos, sendo importante a hidratação adequada dos pacientes antes do início da terapia (Pouchelon, 2015). A desidratação ativa mecanismos compensatórios que aumentam a produção local de angiotensina II, conhecida pelo seu efeito vasoconstritor preferencial na arteriola renal eferente e portanto, um importante fator na manutenção da TFG (Atkins et al., 2002).

No intuito de diminuir a incidência de disfunção renal recomenda-se iniciar os iECAs em baixas doses, monitorando o estado de hidratação do paciente e seu respectivo índice de creatinina, pois normalmente estão associados a um ligeiro aumento da creatinina sérica (cerca de 20\%) em virtude da diminuição temporária da TFG (Carubelli et al., 2018). Em humanos, as diretrizes europeias sugerem que um aumento da creatinina de até $50 \%$ acima da linha de base é aceitável. Se a creatinina elevar-se mais do que $100 \%$ da linha de base a terapia deve ser interrompida (Ponikowski et al., 2016).

\section{Nutrição no Controle Urêmico}

$\mathrm{O}$ uso da dieta renal é uma intervenção terapêutica que aumenta a sobrevida e a qualidade de vida dos pacientes com DRC em função da redução de proteína, fósforo e sódio, e aumento dos valores de vitamina $\mathrm{B}$, fibra solúvel $\mathrm{e}$ densidade calórica, além da suplementação de ômega-3 e adição de antioxidantes. As dietas renais felinas são suplementadas com potássio (Polzin, 2013).

Os sinais gastrointestinais decorrentes da uremia podem ser controlados com bloqueadores de receptores de histamina $\mathrm{H}_{2}$, como a ranitidina (1-2 mg/kg, a cada 8 ou 12 horas, por via oral ou subcutânea) e famotidina ( $1,1 \mathrm{mg} / \mathrm{kg}$, a cada $12 \mathrm{ou}$ 24 horas, por via oral ou subcutânea); e com os inibidores da bomba de prótons de hidrogênio $\left(\mathrm{H}^{+}\right)$gástrica, como o omeprazol $(0,7-1 \mathrm{mg} / \mathrm{kg}$, a cada 12 ou 24 horas, via oral ou intravenosa). Os antieméticos como metoclopramida (0,1-0,5 $\mathrm{mg} / \mathrm{kg}$, a cada 6 ou 8 horas, por via oral ou subcutânea), ondansetrona $(0,5-1 \mathrm{mg} / \mathrm{kg}$, a cada 12 ou 24 horas, por via oral, subcutânea ou intravenosa), bromoprida $(0,2-0,5 \mathrm{mg} / \mathrm{kg}$, a cada 6 ou 8 horas, por via oral), citrato de maropitant (1$8 \mathrm{mg} / \mathrm{kg}$, a cada 24 horas, por via oral ou subcutânea, não recomendada por mais de 5 dias) e protetores de mucosa como o sucralfato (cães:
0,5-2,0 g, a cada 6 ou 12 horas, por via oral; gatos: $0,25-0,5 \mathrm{~g}$, a cada 6 ou 12 horas, por via oral) também são recomendados. Os estimulantes de apetite, como a mirtazapina (cães: $15-30 \mathrm{mg}$ por dia, a cada 24 horas, por via oral; gatos: 1,875-3,75 mg por dia, a cada 48 ou 72 horas, por via oral) e a ciproheptadina (2-4 mg/gato e 5-20 $\mathrm{mg} / \mathrm{cão}$, a cada 8 ou 12 horas, por via oral) podem ser usados na tentativa de aumentar a ingestão de alimentos (Bartges, 2012).

Entretanto, quando a anorexia ou a hiporexia estão presentes, a nutrição enteral pode ser fornecida por meio de sonda nasoesofágica, nasogástrica, esofágica ou gástrica (Polzin, 2013). Nos casos de animais que não toleram nutrientes entéricos e apresentam vômito persistente, a nutrição parenteral pode ser necessária (Langston, 2017).

\section{Tratamento da Anemia}

A gravidade da anemia em cães e gatos com DRC se correlaciona positivamente com as concentrações séricas de creatinina, e a anemia clinicamente relevante é característica de pacientes em estágios IRIS 3-4 da DRC. Além de contribuir para a letargia, anorexia e fraqueza, a anemia grave pode exacerbar a progressão da DRC devido à diminuição da oferta de oxigênio para o rim residual (Fiocchi et al., 2017).

$\mathrm{O}$ tratamento inclui manter um bom estado nutricional, minimizar a perda de sangue gastrointestinal e estimular a produção de glóbulos vermelhos (Bartges, 2012). Medidas terapêuticas são indicadas quando o hematócrito for inferior a $20 \%$, com a utilização de estimuladores da eritropoiese como a eritropoetina humana recombinante $(50$ a $100 \mathrm{UI} / \mathrm{kg}$ de duas a três vezes por semana) e a darbopoetina alfa (1 $\mu \mathrm{g} / \mathrm{Kg}$ a cada 7 dias) (Chalhoub et al., 2012).

\section{Considerações Finais}

O aumento da sobrevida de cães e gatos cardiopatas, em razão dos avanços da terapêutica cardiovascular, deve ser correlacionado com a evolução da SCR. É de extrema importância realizar o estadiamento dos animais com essa síndrome, para que seja definido o tratamento adequado para cada paciente individualmente. Independente da terapia preconizada para estes animais, é recomendada a avaliação renal constante, além de exames de imagens, urinálise, hemograma e hemogasometria, que devem ser considerados em cada caso particularmente. 
Devido à complexidade da síndrome, torna-se essencial o seu diagnóstico precoce e a compreensão de seus mecanismos fisiopatológicos, tendo em vista a minimização de danos dos sistemas cardiorrenais, promovendo maior qualidade de vida e longevidade dos pacientes acometidos.

\section{Conflito de Interesse}

Os autores declaram não existir conflito de interesse.

\section{Comitê de Ética}

O projeto de pesquisa foi aprovado pelo comitê de ética da Universidade de Marília, sob o número 17/2017.

\section{Referências}

Acierno, M.J.; Brown, S.; Coleman, A.E.; Jepson, R.E.; Papich, M.; Stepien, R.L.; Syme, H.M. ACVIM consensus statement: Guidelines for the identification, evaluation, and management of systemic hypertension in dogs and cats. Journal of Veterinary Internal Medicine, 32(6): 1803-1822, 2018.

Atkins, C.; Brown, W.A.; Coats, J.R.; Crawford, M.A.; DeFrancesco, T.C.; Edwards, J.; Fox, J.P.R.; Kenee, B.W.; Lehmkuhl, L.; Luethy, M.; Meurs, K.; Petrie, J.P.; Pipers, F.; Rosenthal, S.; Sidley, J.A.; Straus, J. Effects of long-term administration of enalapril on clinical indicators of renal function in dogs with compensated mitral regurgitation. Journal of the American Veterinary Medical Association, 221(5): 654-658, 2002.

Atkins, C.; Bonagura, J.; Ettinger, S.; Fox, P.; Gordon, S.; Haggstrom, R.; Hamlin, R.; Keene, B.; Luis-Fuentes, V.; Stepien, R. Guidelines for the diagnosis and treatment of canine chronic valvular heart disease. Journal of Veterinary Internal Medicine, 23(9): 1142-1150, 2009.

Bartges, J.W. Chronic kidney disease in dogs and cats. The Veterinary Clinics of North America: Small Animal Practice, 42(4): 669-92, 2012.

Bonagura, J.D.; Bussadori, C.; Church, D. Recommendations for the diagnosis of heart disease and the treatment of heart failure in small animals. In: Miller, M.S.; Tilley, L.P.W.B. Manual of canine and feline cardiology. Philadelphia: Saunders, 1995. p. 469-490.

Bragato, N.; Borges, N.C.; Fioravanti, M.C.S. Bmode and Doppler ultrasound of chronic kidney disease in dogs and cats. Veterinary Research Communications, 41(4): 307-315, 2017.

Carubelli, V.; Metra, M.; Lund, L.H. Negotiating renal dysfunction when treating patients with heart failure. Expert Review of Cardiovascular Therapy, 16(2): 113-122, 2018.

Chalhoub, S.; Langston, C.E.; Farrelly, J. The use of darbepoetin to stimulate erythropoiesis in anemia of chronic kidney disease in cats: 25 cases. Journal of Veterinary Internal Medicine, 26(6): 363-369, 2012.

Chamas, P.P.; Saldanha, I.R.; Costa, R.L. Prevalência da doença degenerativa valvar crônica mitral em cães. Journal of the Health Sciences Institute, 29(3): 214-217, 2011.

Chawla, L.S.; Herzog, C.A.; Costanzol, M.R.; Tumlin. J.; Kellum, J.; McCullough, P.; Ronco, C. Proposal for a functional classification system of heart failure in patients with end-stage renal disease: Proceedings of the Acute Dialysis Quality Initiative (ADQI) XI workgroup. Journal of the American College of Cardiology, 63(13): 1246-1252, 2014.

Davis, H.; Jensen, T.; Johnson, A.; Knowles, P.; Meyer, R.; Rucinsky, R.; Shafford, H. 2013 AAHA/AAFP fluid therapy guidelines for dogs and cats. Journal of the American Animal Hospital Association, 49(10): 149159, 2013.

Dutton, E.; López-Alvarez; J. An update on canine cardiomyopathies - is it all in the genes? Journal of Small Animal Practice, 59 (11): 455-464, 2018.

Feijó, D.V.S.; Finato, R.B.; Fernandez, S.; Dan De Nardo, C.D.; Salvador, R.L.C.; Galvão, A.L.B. Diagnóstico e opções terapêuticas no controle da hipertensão arterial sistêmica em pequenos animais. Investigação, 15(1): 2636, 2016.

Ferasin, L.; Sturgess, C.P.; Cannon, M.J.; Caney, S.M.; Gruffydd-Jones, T.J.; Wotton, P.R. Feline idiopathic cardiomyopathy: a retrospective study of 106 cats (1994-2001). Journal of Feline Medicine and Surgery, 5(8): 151-159, 2003. 
Fiocchi, E.H.; Cowgill, L.D.; Brown, D.C.; Markovich, J.E.; Tucker, S.; Labato, M.A.; Callan, M.B. The use of darbapoetin to stimulate erythropoiesis in the treatment of anemia of chronic kidney disease in dogs. Journal of Veterinary Internal Medicine, 31(2): 476-485, 2017.

Freitas, G.C.; Veado, J.C.; Carregaro, A.B. Testes de avaliação de injúria renal precoce em cães e gatos. Semina: Ciências Agrárias, 35(1): 411-426, 2014.

Freitas, R.A.; Silva B.S.; Athar, C.V.; Marinho J.P.; Veiga, C.C.; Paiva, J.P. Estadiamento da doença renal crônica em cães domésticos com doença valvar crônica. Revista Brasileira de Medicina Veterinária, 38(2): 31-39, 2016.

Grande, D.; Gioia, M.I.; Terlizzese, P.; Iacoviello, M. Heart Failure and Kidney Disease. Advances in Experimental Medicine and Biology, 1067(19): 219-238, 2017.

Grauer, G.F. Diagnosis, management of hypertension, proteinuria in dogs with chronic renal disease. DVM Newsmagazine, 2009. Disponível em: $<$ http://veterinarynews.dvm360.com/diagnosi s-management-hypertension-proteinuriadogs-with-chronic-kidney-disease>. Acesso em: 10 abr. 2018.

Hall, J.A.; Yerramilli, M.; Obare, E.; Yerramilli, M.; Almes, K.; Jewell, D.E. Serum concentrations of Symmetric Dimethylarginine and creatinine in dogs with naturally occurring chronic kidney disease. Journal of Veterinary Internal Medicine, 30(3): 794-802, 2016.

Hsueh, C.; Chen, N.; Lin, S.; Chen, P.; Gattone, V.; Allen, M.; Fishbein, M.; Moe, S. Pathogenesis of Arrhythmias in a Model of CKD. American Society of Nephrology, 25(12): 2812-2821, 2014.

IRIS. International Renal Interest Society. Staging Chronic Kidney Disease (CKD). $2013 . \quad$ Disponível em: $<$ http://www.iriskidney.com/pdf/IRISposter.pd f >. Acesso em: 14 Jul. 2018.

Jacob, F.; Polzin, D.J.; Osborne, C.A.; Neaton, J.D.; Lekcharoensuk, C.; Allen, T.A.; Kirk, C.A.; Swanson, L.L. Association between initial systolic blood pressure and risk of developing a uremic crisis or of dying in dogs with chronic renal failure. Journal of the
American Veterinary Medical Association, 222(3): 322-329, 2003.

Jois, P.; Mebazaa, A. Cardio-renal syndrome type 2: epidemiology, pathophysiology, and treatment. Seminars in Nephrology, 32(1): 26-30, 2012.

Langston, C. Managing fluid and electrolyte disorders in kidney disease. Veterinary Clinics of North America: Small Animal Practice, 47(2): 471-490, 2017.

Lee, Y.J.; Hu, Y.Y.; Lin, Y.S, Wong, M.; KuoHsuan, H.; Hsu, W. Urine neutrophil gelatinase-associated lipocalin (NGAL) as a biomarker for acute canine kidney injury. BMC Veterinary Research, 8(248): 1-9, 2012.

Martins, H.; Pedro, N.; Castellano, M., Monteiro, P., Moura, J.J., Providência, L.A. Síndrome cardio-renal: Os desafios no tratamento da insuficiência cardíaca. Acta Médica Portuguesa, 24(7): 285-292, 2011.

Medeiros, A; Bacurau, A.V.N.; Vanzelli, A.S.; Rolim, N.P.L.; Brum, P.C. Sistema nervoso simpático na insuficiência cardíaca: ênfase na sinalização adrenérgica cardíaca. Revista Hipertensão, 12(3): 84-94, 2009.

Mora, S.D.; Dávila, E.Z.; Silva, E.N.; Mesquita, E.T., Martins, W.A.; Junior, H.V. Síndrome cardiorrenal tipo 1: Mecanismos fisiopatológicos e papel dos novos biomarcadores. Insuficiência Cardíaca, 11(1): 23-30, 2016.

Oliveira, P.; Domenech, O.; Silva, J.; Vannini, S.; Bussadori, R.; Bussadori, C. Retrospective Review of Congenital Heart Disease in 976 Dogs. Journal of Veterinary Internal Medicine, 25(3): 477-483, 2011.

Oliveira, F.M.; Santos, E.A.; Reis, A.C.; Feitosa, C.S.; Cerqueira, H.D.B.; Aptekmann, K.P. Ecocardiografia em cães com doença crônica de valva mitral: revisão de literatura. Tópicos Especiais em Ciência Animal, 6(11): 158171, 2017.

Orvalho, J.S.; Cowgill, L.D. Cardiorenal syndrome: diagnosis and management. Veterinary Clinics of North America: Small Animal Practice, 47(5): 1083-1102, 2017.

Palazzuoli, A.; Ronco, C. Cardio-renal syndrome: An entity cardiologists and nephrologists should be dealing with collegially. Heart Failure Reviews, 16(6): 503-508, 2011.

Palazzuoli, A.; Mccullough, P.A.; Ronco, C.; Nuti, R. Kidney disease in heart failure: the 
importance of novel biomarkers for type 1 cardio-renal syndrome detection. Internal and Emergency Medicine, 10(5): 543-554, 2015.

Peddle, G.D.; Singletary, G.E.; Reynolds, C.A.; Trafny, D.J.; Machen, M.C.; Oyama, M.A. Effect of torsemide and furosemide on clinical, laboratory, radiographic and quality of life variables in dogs with heart failure secondary to mitral valve disease. Journal of Veterinary Cardiology, 14(1): 253-259, 2012.

Polzin, D.J. Evidence-based step-wise approach to managing chronic kidney disease in dogs and cats. Journal of Veterinary Emergency and Critical Care, 23(2): 205-215, 2013.

Ponikowski, P.; Voors, A.; Anker, S.; Bueno, H.; Cleland, J.; Coats, A.; Falk, V.; GonzálezJuanatey, J.; Harjola, P.; Jankowska, E.; Jessup, M.; Linde, C.; Nihoyannopoulos, P.; Parissis, J.; Pieske, B.; Riley, J.; Rosano, G.; Ruilope, L.; Ruschitzka, F.; Rutten, F.; Van der Meer, P. 2016 ESC Guidelines for the diagnosis and treatment of acute and chronic heart failure: the task force for the diagnosis and treatment of acute and chronic heart failure of the European Society of Cardiology (ESC). Developed with the special contribution of the Heart Failure Association (HFA) of the ESC. European Journal of Heart Failure, 37(27): 2129-2200, 2016.

Pouchelon, J.; Atkins, C.; Bussadori, C.; Oyama, M.; Vaden. S.; Bonagura, J.; Chetboul, V.; Elliot, J.; Francey, T.; Grauer, G.; Fuentes, V.; Moise, N.; Polzin, D.; Van Dongen, A.; Van

Shah, B.N.; Greaves, K. The cardiorenal syndrome: a review. International Journal of Nephrology, 2011(11): 1-11, 2011.

Shrestha, H.K.; Beg, M.A.; Siddiqui, M.A.R. Dynamic progesterone responses to stimulation of a natural pulse of a metabolite of prostaglandin $\mathrm{F} 2 \alpha$ in heifers. Animal Reproduction Science, 118(5): 118-123, 2010.

Souza, W.N.; Araújo, C.M.; Silva, S.A.; Petribú, M.M. Anemia, disfunção renal e desnutrição associadas à insuficiência cardíaca em pacientes valvopatas. Arquivos Brasileiros de Cardiologia, 94(6): 794-798, 2010.
Israel, N. Cardiovascular-renal axis disorders in the domestic dog and cat: a veterinary consensus statement. Journal of Small Animal Practice, 56(9): 537-552, 2015.

Prowle, J.R.; Bellomo, R. Fluid administration and the kidney. Current Opinion in Critical Care, 19(4): 308-314, 2013.

Rossi, G.; Bertazzolo, W.; Binnella, M.; Scarpa, P.; Paltrinieri, S. Measurement of proteinuria in dogs: analytic and diagnostic differences using 2 laboratory methods. Veterinary Clinical Pathology, 45(3): 450-458, 2016.

Ronco, C.; Mccullough, P.; Anker, S.D.; Anand, I.; Aspromonte, N.; Bagshaw, S.; Bellomo, R.; Berl, T.; Bobek, I.; Cruz, D.; Daliento, L.; Davenport, A.; Haapio, M.; Hillege, H.; House, A.; Katz, N.; Maisel, A.; Mankad, S., Zanco, P.; Mebazaa, A.; Palazzuoli, A.; Ronco, F.; Shaw, A.; Sheinfeld, G.; Soni, S.; Vescovo, G.; Zamperetti, N.; Ponikowski, P. Cardiorenal Syndromes: report from the consensus conference of the Acute Dialysis Quality Initiative. European Heart Journal, 31(6): 703-711, 2010.

Ronco, C.; Di Lullo, L. Cardiorenal syndrome in western countries: Epidemiology, diagnosis and management approaches. Kidney Diseases, 2(4): 151-163, 2017.

Sarraf, M; Masoumi, A; Schrier, R.W. Cardiorenal Syndrome in Acute Decompensated Heart Failure. Journal of the American Society of Nephrology, 4(12): 2013-2026, 2009.

Strunz, C.M.; Marcondes-Santos, M.; Takada, J.Y.; Fragata, F.S.; Mansur, A.P. Escore de qualidade de vida como preditor de mortalidade em cães com doença degenerativa da valva mitral. Arquivos Brasileiros de Cardiologia, 108(7): 347-353, 2017.

Tidholm, A.; Ljungvall, I.; Michal, J.; Haggstrom, J.; Hoglund, K. Congenital heart defects in cats: a retrospective study of 162 cats (1996 e 2013). Journal of Veterinary Cardiology, 17(4): 215-219, 2015.

Yu, I.B.; Huang, H.P. Prevalence and prognosis of anemia in dogs with degenerative mitral valve disease. BioMed Research International, 2016(5): 1-5, 2016. 